Review Article

\title{
Critical review of clinical trials in senile dementia $-I^{*}$
}

\author{
J.M. Orgogozo ${ }^{1}$ and R. Spiegel ${ }^{2}$
}

${ }^{\prime}$ Neurological Department, University of Bordeaux II, Pellegrin Hospital, F-33076, Bordeaux Cedex, France and ${ }^{2}$ Clinical Research, CNS-Psychogeriatrics, Sandoz Ltd., CH-4002 Basle, Switzerland

\section{Introduction}

Three major problems are encountered in carrying out a literature survey on clinical trials of treatments for senile dementia: (i) the reviewer might become discouraged by the fact that hopes raised by anecdotal observations or preliminary clinical trials are nearly always dashed by subsequent papers contradicting the early findings. Obviously, the anxiety engendered by senile dementia and the publicity which it attracts predispose to premature publication of preliminary results. (ii) By their very nature, treatments which are essentially empirical lack credibility. Most of the 'treatments' advocated today were developed before the present-day hypotheses on the pathophysiology of senile dementia had been put forward, but some of these treatments have subsequently been found to tie in with one or more of these pathophysiological hypotheses. Nevertheless, these hypotheses throw light on the cause of the disease: the abnormal enzyme activity and neurotransmitter levels described some years ago ${ }^{1}$ may just as well be a consequence of the disease as a cause and hence the pathogenesis and origin of primary degenerative dementia (PDD) remain to all intents and purposes a mystery. (iii) It would be tempting to look for errors or methodological shortcomings in the scores of publications which appear every year, the aim being to prove that the trials are so badly flawed that no conclusions can be drawn from them. Such a malevolent attitude might be appropriate for positive trials if they are of implicitly low credibility and demonstrate only a small effect or effects which are not reproducible. However, negative trials are often inconclusive because their samples are too small to yield statistically significant results.

Correspondence: Professor J.M. Orgogozo M.D.

Accepted: 12 November 1986

* Part II of this review, together with the references, will be published in the May 1987 issue.
We have analysed mainly general reviews and papers on the subject appearing in the past ten years since, before 1975 , most of the trials were carried out in geriatric patients with poorly defined disorders or with conditions described as "cerebrovascular disease or insufficiency'. Only since 1975 have most studies been conducted in patients whose clinical signs and symptoms are compatible with the diagnosis of primary degenerative dementia (PDD). Latterly, more precise diagnostic criteria for PDD have been proposed in the DSM III ${ }^{2}$ and for Alzheimer's disease by the NINCDS-ADRA workgroup. ${ }^{3}$ Our aim has been to trace the development of some new ideas about investigational drugs, whilst confining ourselves to the treatment of PDD and related conditions, and analysing only those pilot studies, controlled clinical trials and reviews which allow at least a partial assessment of the methodology used.

Since the criteria for patient selection and evaluation of therapeutic efficacy have been treated in some previous reviews ${ }^{4,5}$ we shall concentrate mainly on the effect of the various drugs which have been advocated for treatment of PDD and also for impairment of memory occurring in isolation. The latter is regarded, either as a model, or as an early form of PDD. It should however be mentioned in this preamble that the greatest obstacle to interpreting the results of even recent trials is a lack of precision concerning the diagnostic criteria for PDD. Furthermore, bias shown by journals in the selection of papers favours studies on those drugs which have been most extensively tested: a paper reporting positive results has a better chance of being accepted than a paper reporting a negative outcome, this apart from the 'censorship' exercised by investigators and pharmaceutical companies, who may have less motive for submitting negative trials for publication.

Lastly, it should be borne in mind that many of the drugs described below and grouped according to a simple classification ${ }^{6}$ possess more than one phar-

The Fellowship of Postgraduate Medicine 1987 
macological mode of action, that the mode of action attributed to one and the same drug may have changed over the years and that the relation between pharmacological action and clinical efficacy remains in doubt in most cases.

\section{Vasodilators}

The cerebral vasodilators are commonly, but rather arbitrarily, sub-divided into two classes: ${ }^{4}$ those with a pure vasodilator action and those with vasodilator and metabolic effects. The rationale behind their use was the idea, now considered erroneous, that most, if not all, cases of senile dementia were due to 'cerebrovascular insufficiency'. The cerebral vasodilator effect of most of these drugs has since been either invalidated or relegated to second place, emphasis now being laid on other modes of action, involving various levels of energy metabolism or drug effects on neurotransmitters.

The majority of drug trials based on the original concept date from the 1960s and 1970s and have been the subject of numerous reviews, notably by Prien, ${ }^{7}$ Hollister, ${ }^{8}$ Gaitz et al., ${ }^{9}$ Yesavage et al..${ }^{4,10}$ Reisberg et al., ${ }^{11}$ and Cook \& James. ${ }^{12}$ Many of them do not meet present-day methodological standards with regard to one or more of the following criteria: (1) Patient selection (poorly defined samples, inclusion of patients suffering from 'cerebrovascular insufficiency' or 'vascular dementia', without employing criteria to distinguish it from the most common form of dementia, i.e. Alzheimer's disease); (2) Trial design (more than half were open, descriptive studies, not taking into account the possibility of time-related and placebo responses).

Such limitations almost completely invalidate the positive results, i.e. improvement of performance in psychometric tests or in overall clinical status, reported in a number of these trials. The subsequent loss of credibility, coupled with disappointed clinical impressions of prescribers, has meant that most of the socalled 'pure' cerebral vasodilators have found less and less application in PDD.

Cyclandelate has been the subject of a series of studies which have yielded equivocal results (see detailed analysis by Wittenborn ${ }^{5}$ ). Among the more recent papers, the one by Rao et al. ${ }^{13}$ showed that the drug had a definite but inconsistent effect in patients with senile mental changes, and Capote \& Parikh ${ }^{14}$ concluded that cyclandelate was a moderately effective and safe treatment for some symptoms of senility in carefully selected patients; unfortunately, the patients recruited for their study were selected only on the basis of age (over 65 years of age) and long-term hospitalization.

Of eight early trials of naftidrofuryl reviewed by Yesavage et al., ${ }^{10}$ seven were considered to be of a suitable methodological standard, and the authors concluded that the drug had shown efficacy in all of $\overline{3}$ them. This statement must be qualified in the light of $\stackrel{\mathbb{Q}}{\varrho}$ an analysis of three publications which appeared in $C$ leading journals: while $\operatorname{Cox}^{15}$ found the drug to be $\overrightarrow{\bar{F}}$ effective in elderly confused patients, he acknowledged that its effects were very variable and that there was great inter-observer variation. Brodie ${ }^{16}$ studied deteriorated elderly patients by means of simple criteria and tests and concluded that there was a limited but measurable improvement in daily living of activities, but no effect on intellectual performance; the drug produced few side effects. Branconnier \& Cole $^{17}$ studied 60 patients suffering from early PDD using a sophisticated test battery. In this double-blind trial in parallel groups, the group treated with naftidrofuryl showed significant improvement in reaction time, short-term memory and visual memory, but performance was improved in only 5 of the 21 tests, and, more important, there was no overall improvement in behaviour.

Co-dergocrine mesylate is almost in a class of its own, since it has been the subject of a larger number of controlled clinical trials than any other drug, including some conducted in the $1980 \mathrm{~s}$, and since these trials have contributed to the development of improved methods of drug testing in psychogeriatrics. A number of authors ${ }^{18-22}$ have reviewed the papers published prior to 1980, beginning with the well-conducted triat by Rosen, ${ }^{23}$ who concluded that co-dergocrine mesylate was superior to papaverine in mental decline in the elderly.

Most of the older controlled trials with co-dergocrine mesylate showed a statistically significant improvement in some items of the Sandoz Clinical Assessment Geriatric (SCAG) Scale, ${ }^{24,25}$ particularly in the emotional and cognitive variables. Methodological objections frequently raised against these positive results relate to bias in the selection of articles for publication by the medical journals which has already been mentioned, and to the statistical shortcomings inherent in multiple-item rating scales and the lack of formal validation of this type of scale: (1) statistical problems associated with rating scales have to do with the fact that such instruments usually comprise a large number of items, and this increases the probability of a type I statistical error: with a scale of 10 items there is a $1: 2$ chance of a fortuitous improvement in a single item at the $5 \%$ level; (2) rating scales ought to be validated by comparing the scores (and the variation in scores) with the 'actual' clinical status, i.e. patients' ability to lead an independent life, and their motor, intellectual and communicative performance, or indeed their life expectancy and quality of life. No such validation has as yet been made.

However, these general problems do not invalidate all the positive results, and the following observations 
bear out the fact that co-dergocrine mesylate is effective in PDD (even though its efficacy is limited): (1) an increase in therapeutic effect is observed with prolonged treatment; ${ }^{26,27}(2)$ increased efficacy is seen at higher doses of 6 or even $9 \mathrm{mg} /$ day instead of the usual 3 or $4.5 \mathrm{mg} ;{ }^{28,29}$ (3) the scores for the items of the SCAG scale most frequently improved, namely 'impairment of recent memory', 'disorientation', 'confusion', 'mental alertness' and 'depression', are

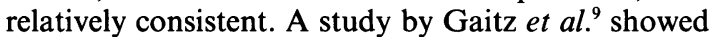
that the effects on mental deterioration were independent of a predominant antidepressant effect.

A retrospective analysis by Singer et al. ${ }^{30}$ concluded that the drug was more effective in PDD than in arteriosclerotic dementia (MID), but the data presented do not seem to us too convincing, since the item 'overall impression of patient' was improved more frequently in patients with MID. Hollister \& Yesavage $^{31}$ stated that the data from the literature confirmed the short-term efficacy of the drug in PDD and, that of the treatments currently available, dihydroergotoxine would be one of the best candidates for a long-term trial.

Few trials have been carried out on ginkgo biloba extract, a product recently introduced in some European countries for use in this indication. In normal subjects it appears to be capable of improving performance in some memory tests, ${ }^{32}$ from a preliminary controlled trial in a small number of very old patients, it was concluded that the drug improved the quantified electroencephalogram (EEG) and also the clinical status, as assessed on the SCAG scale. ${ }^{33}$ A larger controlled parallel-group trial in 40 patients using well-defined criteria for early PDD indicated that there was an overall subjective improvement and better performance in various psychometric and behavioural tests, the improvement being greater after 12 weeks than after 8 weeks of treatment. ${ }^{34}$ Here too, of course, the same reservations apply concerning the validity of rating scales and bias in the selection of papers for publication.

\section{Drugs with metabolic activity}

One of the most widely used and most frequently investigated drugs is piracetam..$^{5,6,22,35}$ Of the various treatments having an exclusively metabolic action, hyperbaric oxygen therapy may be considered to be a model, while pyritinol, meclofenoxate and almitrine in combination with raubasine may be regarded as practical applications.

The mode of action of piracetam is poorly understood, and its use in the treatment of PDD was suggested by its behavioural effects in laboratory animals. Demonstration in animal experiments that it facilitated transcallosal synaptic transmission ${ }^{36}$ led to the concept of 'nootropic' substances which might be of value for the treatment of impairment of intellectual function in the aged. Piracetam improved verbal learning in normal young subjects. ${ }^{37}$ Thirteen controlled clinical studies were reviewed by McDonald. ${ }^{22}$ Eight of these were conducted in parallel groups and the other 5 were crossover studies. Seven of these trials were negative, and this was attributed in some cases to the fact that the patients had deteriorated too far, to an inadequate dosage or to an inadequate period of observation. Nevertheless, some of these negative trials were carried out in patients with early dementia who received a high dosage (up to $9.6 \mathrm{~g}$ /day) for periods of up to three months. This period of observation may still be too short, since it is today recommended that patients be observed for at least 6 months and if possible for one year. ${ }^{38}$ In the 6 positive trials significant effects were reported four times, mainly for the items 'motor activity', 'cognitive function' and 'mood' and for 'general impression of efficacy'. No definite conclusion can be drawn from these results as to whether the drug is effective to the extent that it may appreciably modify the patient's survival or ability to live an independent life. However, this conclusion has still to be confirmed in further studies, as for the previously mentioned drugs.

Hyperbaric oxygen therapy was found to be effective in some older open studies and in the first controlled trials, ${ }^{39}$ but was abandoned practically everywhere following the trial by Raskin et al ${ }^{40}$ in 82 patients studied under rigorous conditions. These authors concluded that hyperbaric oxygen treatment was ineffective, despite marginal improvements in certain evaluation criteria. This example may serve as a salutary warning, since it is one of the rare cases where a well conducted study was able to put an end to speculation based largely on theoretical considerations and anecdotal reports. The fact that hyperbaric oxygen therapy is expensive and inconvenient may be another factor in the abandonment of this therapy.

Meclofenoxate does not appear to have been investigated in recent controlled trials. After a series of older studies producing conflicting results, a paper appeared by Marcer $\&$ Hopkins ${ }^{41}$ who concluded that the drug was to some extent effective in isolated memory disorders in elderly people.

Pyritinol, like the last-mentioned drug, is said to have an 'anti-anoxic' action, promoting increased glucose utilization even in hypoxaemic states of the brain. It was concluded in two trials ${ }^{42,43}$ that in PDD and vascular dementias the drug afforded marginal improvement, which was not, however, statistically significant. As in numerous other studies the improvement was found to be inconsistent, only a minority of patients benefiting from the treatment, so that the drug is of little practical interest.

Almitrine combined with raubasine has so far been 
the subject of only a few controlled trials. Berthaux \& Laforestrie $^{44}$ tested the drug in 29 patients, selected simply because they were on a geriatric ward and were displaying 'intellectual deterioration'. They found an improvement in reaction time and visual retention (Benton test). Pardo ${ }^{45}$ in a similar study in 40 elderly hospitalized patients found an improvement in the 'Overall Geriatric Evaluation' as well as in various tests. As in the previous trial, however, there is nothing in Pardo's paper to indicate whether these patients met the criteria for PDD. A more detailed study in 50 very old deteriorated patients ${ }^{46}$ investigated the effect of this drug, given for 2 to 7 months in accordance with a double-blind procedure; the results obtained using a behavioural rating scale and two simple psychometric tests were moderately favourable, but no conclusions can be drawn from them since 9 patients, 7 of them in the treated group, were withdrawn from the trial.

\section{Gerovital H3}

The history of gerovital ${ }^{47}$ is an interesting one, illustrating as it does the superiority of correct methodology over therapeutic empiricism: after a period of 25 years which saw the publication of reports of, sometimes spectacular, anecdotal observations and of claims owing more to enthusiasm than to reason, several controlled clinical trials showed that this mixture of analgesic and metabolically active substances was in fact ineffective. ${ }^{48}$ The two positive trials among those published before 1980 were carried out in small series of patients (30 and 31 patients) hospitalized in geriatric departments, either for mental confusion or for depression. Six other studies carried out in the same kind of patients were all negative. ${ }^{5}$

\section{Psychostimulants}

The hypothesis that a primary deficit of 'attention', 'interest', or vigilance in the general sense is responsible, in part at least, for the other intellectual or behavioural deficits of elderly deteriorated persons has prompted the use of psychostimulants in an attempt to 'activate' these patients. And, indeed, such

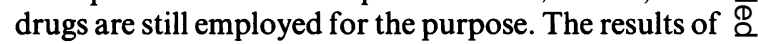
clinical trials may be summed up by stating that any $C$ benefits are inconsistent and not very convincing, and $\vec{F}$ that side effects such as confusion and agitation are common.

Of the drugs which have been the subject of controlled trials, one of the most thoroughly investigated is methyl-phenidate. On the whole the results have been judged negative, ${ }^{49,50}$ although instances of $\nRightarrow$ 'overall general improvement ${ }^{\text {s1 }}$ or improvement in certain cognitive tests ${ }^{52}$ have been reported.

Pentylenetetrazole, which has also been extensively investigated, gave negative results in 8 out of 13 trials reviewed by Prien $^{7}$ and did not afford improvement in cognitive function in any of the 16 controlled trials reviewed by Loew \& Singer; ${ }^{50}$ on the other hand, behavioural improvement was reported but was not consistently observed.

Amphetamine and its direct derivatives have such severe cardiovascular and cerebral side effects that they should not be employed routinely in geriatric medicine, although they have been tried..$^{53}$ Because of this, only anecdotal observations are available. ${ }^{50}$

Caffeine and theophylline have not, to our knowledge, been investigated in clinical trials propes in this indication; another xanthine base, pento ifylline, has been widely investigated in cerebrovas cular disorders because of its effect on blood flow, but we have only found one controlled trial in an indication related to dementia ('chronic cerebrovascular insufficiency'). The paper reported an improvement in psychometric test results and in such items as 'social isolation', 'general agitation' and 'sleep disturbances'. ${ }^{54}$ A combination of pentroxifylline with piracetam, given orally, seemed to be more efficient than either drug alone in a prospective trial on 80 elderly patients with minor or mild mental deterioration..$^{55}$ However the only striking change was on verbal memory, while piracetam alone seemed to improve mainly visual memory, and pentoxifylline alone had only a haemorheological, but no psychological effect. 